International Mathematical Forum, 2, 2007, no. 29, 1431 - 1437

\title{
Local Connectivity of the Julia Set of Polynomials on the Cone
}

\author{
Ahmad Zireh \\ Department of Mathematics, Shahrood University \\ of Technology, P.O.Box 316-36155 Shahrood, Iran \\ azireh@gmail.com
}

\begin{abstract}
In the dynamics of quadratic polynomials $P_{c}(z)=z^{2}+c$ in the complex plan, Yoccoz has proved that under suitable conditions the Julia set $J\left(P_{c}\right)$ is locally connected. This result has been extended to the dynamics of uni-critical polynomials $z^{d}+c[5]$. In this paper we extend the Yoccoz's Theorem to polynomials on the Cone.
\end{abstract}

Keywords: locally connected, polynomial dynamic, Cone, renormalizable, iteration

Mathematics Subject Classification: Primary 32H50, Secondary 37F45, 30D05

\section{Introduction}

We first recall some terminology and definitions in holomorphic dynamics in the complex plan(see[7]). let $f: \mathbb{C} \rightarrow \mathbb{C}$ be a polynomial self-map of the complex plan. For each $z \in \mathbb{C}$, the orbit of $z$ is

$$
\operatorname{Orb}_{f}(z)=\left\{z, f(z), f(f(z)), \cdots, f^{n}(z), \cdots\right\} .
$$

The dynamical plane $\mathbb{C}$ is decomposed into two complementary sets: the filled Julia set

$$
K(f)=\left\{c \in \mathbb{C}: \quad \operatorname{Orb}_{f}(z) \text { is bounded }\right\},
$$

and its complementary, the basin of infinity

$$
A_{f}(\infty)=\mathbb{C}-K(f) .
$$

The boundary of $K(f)$, called the Julia set, is denoted by $J(f)$.

When $f$ is a quadratic polynomial, say $f(z)=P_{c}=z^{2}+c$, in the extended 
complex plane $\hat{\mathbb{C}}=\mathbb{C} \cup\{\infty\}$, the point at infinity is a super-attracting fixed point and hence there exists $U_{c}$ a neighborhood of infinity and a conformal map(the Bottcher map),

$$
\varphi_{c}: U_{c} \rightarrow\{z \in \mathbb{C} ;|z|>1\}
$$

such that $\varphi_{c}(\infty)=\infty, \varphi_{c}^{\prime}(\infty)=1$ and $\varphi_{c} \circ P_{c}=\left(\varphi_{c}\right)^{2}$. On the other hand, if the critical point 0 is in $K\left(P_{c}\right)$, then $K\left(P_{c}\right)$ is connected otherwise $K\left(P_{c}\right)$ is totally disconnected.

The Mandelbrot set $\mathcal{M}_{2}$ is defined as the set of parameter value $c$, for which $K\left(P_{c}\right)$ is connected

$$
\mathcal{M}_{2}=\left\{c \in \mathbb{C}: K\left(P_{c}\right) \text { is connected }\right\} .
$$

From Yoccoz's Theorem, $M_{2}$ is locally connected for all parameters in the boundary of hyperbolic componets $M_{2}[4]$. In the last decades, the topological and measure theoretical properties of these sets have been extensively studied. Using techniques and notions based on the quasi-conformal surgery, polynomial-like map, renormalizability, Yoccoz puzzle, Hubbard's tableau, etc., the following substantial result have been obtained:

Yoccoz Theorem.([4]) If $P_{c}$ has no indifferent periodic points and is not infinitely renormalizable, then the Julia set $J\left(P_{c}\right)$ is locally connected.

The extension of this theorem to other classes of polynomials constitutes part of today's redsearch in this area. For instance, Yoccoz Theorem have been extended to uni-critical polynomials $g_{c}(z)=z^{d}+c($ see[5]).

We would like to study dynamics of maps in other domains, in special case, dynamics the polynomials on the Cone. We want to extend Yoccoz Theorem to the class of polynomials on the Cone.

Our main result now reads as:

Main result.(Extension of Yoccoz's Theorem)If the polynomial on the Cone, $\tilde{f}_{c}(z)$, is not infinitly renormalizable and does not have an indifferent periodic point then its julia set $J\left(\tilde{f}_{c}\right)$ is locally connected.

\section{Polynomials on the Cone.}

In this section we consider a condition for family of polynomials. Milnor[7] introduced a one-parameter family $f(z)=k\left(z+z^{-1}\right)$ of quadratic rational maps. We wanted to reduce dependency polynomial $f(z)$ from $(d-1)$ parameter to one parameter. For this reason, in analogue to [7] we define an 
automorphism of a complex polynomial $f(z)=z^{d}+a_{d-2} z^{d-2}+\cdots+a_{0}$ a linear map $R(z)=a z+b ; \quad a, b \in \mathbf{C}$. Which commutes with $f$ so that $R \circ f \circ R^{-1}=f$. The collection of all automorphisms Aut $(f)$ forms a finite subgroup of rotation group $\operatorname{Rot}_{d}(f)=\left\{a \in \mathbf{C} ; a^{d-1}=1\right\}$.

In special case two groups $A u t(f)$ and $\operatorname{Rot}_{d}(f)$ are equal. Hence we have a trivial Lemma.

Definition 2.1 Let $R(z)=e^{2 \pi i / d} z$. With the equivalence relation $z \sim z^{\prime} \Leftrightarrow$ $\exists n ; z=R^{n}\left(z^{\prime}\right)$. Denote $\tilde{\mathbf{C}}$ the set of all equivalent class in $\mathbf{C}$ (that is homeomorphic to cone), and $\tilde{f}: \tilde{\mathbf{C}} \rightarrow \tilde{\mathbf{C}}$ the complex polynomials on Cone, $\tilde{\mathbf{C}}$ (induced by polynomial $f$ under $\sim$ ).

Lemma 2.2 A monic centered polynomial $\tilde{f}(z)$ of degree d possesses a nontrivial automorphism equal to $\operatorname{Rot}_{d}(f)$ if and only if it is conjugate to a form $\tilde{f}_{c}(z)=z^{d}+c z, c \in \mathbf{C}$.

The polynomial $\tilde{f}_{c}(z)=z^{d}+c z, c \in \mathbf{C}$ has a critical point

$$
\left[c^{0}\right]=\left(\frac{-c}{d+1}\right)^{1 / d}
$$

which is a member of equivalence relation i.e.

$$
\left[c^{0}\right]=\left[c^{1}\right]=\left[\omega c^{0}\right]=\cdots=\left[c^{d-1}\right]=\left[\omega^{d-1} c^{0}\right] \text { such that } \omega=e^{2 \pi i / d},
$$

and a critical value $\tilde{f}_{c}\left(c^{0}\right)$ such that

$$
\left[\tilde{f}_{c}\left(c^{0}\right)\right]=\left[\tilde{f}_{c}\left(c^{1}\right)\right]=\left[\omega \tilde{f}_{c}\left(c^{0}\right)\right]=\ldots=\left[\tilde{f}_{c}\left(c^{d-1}\right)\right]=\left[\omega^{d-1} \tilde{f}_{c}\left(c^{0}\right)\right]
$$

\section{Modified Yoccoz Puzzle}

Fix $d \geq 2$ and a complex polynomial $\tilde{f}_{c}(z)=z^{d}+c z$. If $|c|<1$ then fixed point $z_{0}=0$ is attractive and basin domain of it contains all critical points and then $J(f)$ is Jordan curve [10]. Therefore we consider case $|c|>1$. For $|c|>1$ the fixed point $z_{0}=0$ is repelling. From Douady landing theorem there exist finite external rays $r_{\theta_{i}} ; \quad i=1, \cdots, p$ that land on $z_{0}$. Also from the relation $R \circ f=f \circ R$, if $r_{\theta}$ lands on $z_{0}$ then $r_{\omega \theta}$ lands on $z_{0}$. Therefore $p=q d$, means that the $q$ rays land at $z_{0}$ in space $\tilde{\mathbf{C}}$.

Let $h_{f}$ be the Green's function of $f(z)$ defined on $\mathbf{C} \backslash J$. Since $\widetilde{\mathbf{C} \backslash \mathbf{J}}=\tilde{\mathbf{C}} \backslash \tilde{J}$ denote $h_{\tilde{f}}=\left.h_{f}\right|_{\widetilde{\mathbf{C} \backslash \mathbf{J}}}$ the Green's function defined on $\tilde{\mathbf{C}} \backslash \tilde{\mathbf{J}}$ and choose $R>1$.

Let $\tilde{U}_{0}$ be the region

$$
\tilde{U}_{0}=\left\{z \in \tilde{\mathbf{C}} ; h_{\tilde{f}}(z)<R\right\}
$$


and let $\Gamma_{0} \subset \tilde{U}_{0}$ be the graph formed by the parts of the rays $r_{\theta_{1}}, \ldots, r_{\theta_{q}}$ in $\tilde{U}_{0}$. Then $\Gamma_{0}$ cuts $\tilde{U}_{0}$ into finitely many domains.

Set $\tilde{U}_{n}=\tilde{f}^{-1}\left(\tilde{U}_{n-1}\right)$ and $\Gamma_{n}=\tilde{f}^{-1}\left(\Gamma_{n-1}\right)$. The sequence $\tilde{U}_{0} \supset \tilde{U}_{1} \supset \cdots$ together with the graph $\Gamma_{n} \subset U_{n}$ is called the Yoccoz puzzle $\tilde{P}$ of $\tilde{f}$. The set $\tilde{P}(n)$ of pieces at depth $n$ of the puzzle $\tilde{P}$ is the set of closures of components of $\tilde{U}_{n} \backslash \Gamma_{n}$ in $\tilde{U}_{n}$. An end $x$ of the puzzle $\tilde{P}$ is a nested sequence

$$
x=\left(X_{0} \supset X_{1} \supset \cdots\right)
$$

The domain $C_{n}$ in $\tilde{P}(n)$ containing critical point $c^{0}$ is called critical piece.

\subsection{Tableaux of ends}

By analogue with [4] a tableau $T(x)$ of an end $x$ is two-dimensional array of pieces

$$
T_{n, k}(x)=X_{n}\left(\tilde{f}^{k}(x)\right)
$$

In the tableau the critical positions (the positions for which the corresponding piece contain the critical point $\left.c^{0}\right)$ are interested. A critical position $(n, k)$ will be called strictly critical position if the critical point is in $T_{n+1, k}$ and semicritical position if the critical point is in $T_{n, k} \backslash T_{n+1, k}$. The marked grid is the array with the critical positions marked. The nest of an end $x=\left(X_{0} \supset\right.$ $X_{1} \supset \cdots$ is the sequence of possibility degenerate annuli $A_{1}, A_{2}, A_{3}, \cdots$ where $A_{i}=X_{i}-\operatorname{int}\left(X_{i+1}\right)$. Degenerate annuli occur when $\partial X_{i} \cap \partial X_{i+1} \neq \emptyset$. In this case we set the modulus of the degenerate annulus to be zero. To each end we associate its impression:the compact connected subset

$$
\Im(x)=\bigcap_{n} X_{n}(x) \subset C
$$

As in [4], the reason to introduce nests is the following Lemma.

Lemma 3.1 If for some nest $A_{0}(x), A_{1}(x), \cdots$ the series $\sum_{n=0}^{\infty} \bmod \left(A_{n}\right)$ is divergent, then diam $X_{n} \rightarrow 0$ as $n \rightarrow \infty$, and $\Im(x)$ is a point.

By analogue with[4] we have

Lemma 3.2 The ends of puzzle $\tilde{P}$ have marked grids with following properties:

(a)Every column is either entirely strictly critical, or entirely non-critical, or has a unique semi-critical position, with everything above strictly critical and everything below non-critical.

(b)If the $(n, k)$ position of a marked grid is critical, then $(i, j+k)$ position 
of that marked grid is of the same nature as the $(i, j)$ position of the critical marked grid.

(c)If for the critical grid, the position $(n, k)$ is strictly critical and the positions $(n+i, i)$ aren't critical for $0<i<k$, and if in the tableau $T(x)$ of some end the position $(n+k, l)$ is semi-critical, then the position $(n, l+k)$ of that grid is semi-critical.

An end $x$ of a puzzle $\tilde{P}$ will be called off-critical if there is a critical pieces $C_{N}$ of the critical point such that the orbit of $x$ never enters $C_{N}$.

The polynomial $\tilde{f}$ will be called non-recurrent if the orbit of the critical values never enters $C_{N}$ for some $N$.

Proposition 3.1 . Let the polynomial $\tilde{f}_{c}$ have a critical tableau periodic of least period $k$ and let $N$ be so large that $X_{n, l} \neq C_{n}$ when $n>N$ and $0<l<k$. Then the mapping

$$
\tilde{f}^{k}: C_{N+k} \rightarrow C_{N}
$$

is a quadratic-like map, with connected Julia set. Conversely, if $\tilde{f}$ is $k$-renormalizable and $k$ is the smallest integer with this property, then there exists $N$ such that $\tilde{f}^{k}\left(C_{n}\right)=C_{n-k}$ for all $n>N$.

Proof. From the hypothesis the critical orbit $\left\{\tilde{f}^{k j}\right\}_{j=0}^{\infty}$ remains in $C_{n}$ for every $n$, then from(1) there exists integer $s$ such that the orbit $\left\{f^{s k j}\right\}_{j=0}^{\infty}$ remains in $C_{n}$ for every $n$. Choose $N$ so large that there is not critical or semi-critical annulus of depth $N$. Then for $n>N$ the polynomial-like map $f^{k}: C_{N+k} \rightarrow C_{N}$ has degree $2^{s}$ in fact $s$-times iterate quadratic-like map $\tilde{f}$. For second part, observe that if $\tilde{f}$ is renormalizable, then domain $\tilde{U}$ of the renormalization can be chosen to contain the critical point. Let $K^{\prime}$ be the filled-in Julia set of the renormalized mapping. No preimage of $z_{0}=0$ belong to $K^{\prime}$, unless $0 \in K^{\prime}$. Also $z_{0}$ and inverse images do not disconnect $K^{\prime}$. So $K^{\prime} \subset \bigcap C_{n}$. We choose $N$ so large that $C_{N+k} \subset \tilde{U}$ then we have the result.

The following two proposition are similar to the quadratic case [4].

Proposition 3.2 Let $\tilde{f}$ be non-renormalizable then there exists a smallest integer $m$ such that the entry $(0, m)$ in the critical tableau is the first semicritical entry in the 0 th row, and the annulus at depth $m$ in the critical nest is non-degenerate.

Proposition 3.3 If $\tilde{f}$ is non-recurrent, then all its ends are point, and $J(\tilde{f})$ is locally connected.

A quadratic polynomial with connected Julia set has two fixed points. One of the landing point of the unique fixed ray at angle 0; this point is called the 
$\beta$-ponit. The other fixed point is called the $\alpha$-point. With our assume, $\alpha$ is repelling fixed point hence finite ray at least two land on $\alpha$.

\section{Main Result}

Now we are going to prove the local connectivity theorem for the polynomials on the Cone:

Theorem 4.1 (Extension of Yoccoz's Theorem)If the polynomial on the Cone, $\tilde{f}_{c}(z)$, is not infinitly renormalizable and does not have an indifferent periodic point then its julia set $J\left(\tilde{f}_{c}\right)$ is locally connected.

Proof. The non-renormalizable case proved in [8]. Hence we consider the finitely renormalizable case. Let $\tilde{f}$ be finitely renormalizable polynomial. We will define a new puzzle adapted to the situation. Let $\left.\tilde{f}^{k}\right|_{\tilde{U}}: \tilde{U} \rightarrow \tilde{V}$ be the 'last' renormalization of $\tilde{f}$. From proposition $3.1, \tilde{f}^{k}$ is hybrid equivalent to quadratic polynomial $g(z)=z^{2}+c$. Let $\alpha$ be the fixed point of $\tilde{f}^{k}$ corresponding to the fixed point $\alpha^{\prime}$ of $g$. We define the graph $\Gamma=\Gamma^{\prime} \cup \Gamma^{\prime \prime}$ as follows:

(1) $\Gamma^{\prime}=\Gamma_{0}^{\prime} \cup \ldots \cup \Gamma_{k-1}^{\prime}$ is the union of the external rays of $\tilde{K}$ landing at the points

$$
\alpha, \tilde{f}(\alpha), \cdots, \tilde{f}^{k-1}(\alpha)
$$

$(2) \Gamma^{\prime \prime}=\Gamma_{0}^{\prime \prime} \cup \ldots \cup \Gamma_{l}^{\prime \prime}$ where $\Gamma_{0}^{\prime \prime}$ is the graph formed by the union of the rays landing at the fixed point $z_{0} \cdot \Gamma_{n}^{\prime \prime}=\tilde{f}^{-1}\left(\Gamma_{n-1}^{\prime \prime}\right)$, and $l$ is the smallest number such that one component of $\Gamma_{l}^{\prime \prime}$ separates $\Gamma_{1}^{\prime}$ (the component of $\Gamma_{1}^{\prime}$ associated to the critical value $\left.\tilde{f}\left(c^{0}\right)\right)$ from all other components of $\Gamma^{\prime}$ and $\Gamma_{s}^{\prime \prime}$ with $s<l$. Because of the density of inverse images of $\alpha$, there is such a number $l$.

Restrict the mapping to a region $\tilde{U}$ bounded by some equipotential. The pair $\tilde{U}, \Gamma$ satisfies the conditions of Proposition 11.1 [4]. From the proposition 3.2, every position in the critical tableau in 0th row will be strictly critical until one reaches a first semi-critical position $(0, m)$. The graph carefully chosen so that the non-critical pieces at level 1 contained in the critical piece at level 0 and which intersect $\tilde{K}(\tilde{f})$ are contained in the interior of the critical piece $C_{0}$. Hence the critical piece $C_{m}$ contains the next piece in its interior, giving a non-degenerate annulus in the critical nest. If the tableau is non-recurrent of Proposition 3.3, we have the result. The tableau cannot be periodic, since in that case $\tilde{f}^{k}$ would be renormalizable[proposition 3.1], hence we have the desired result. 


\section{References}

[1] B. Branner and J. H. Hubbard, The iteration of cubic polynomials, Part II:pattern and parapatterns, Acta. Math. 169 (1992), no 3-4, pp 229-325.

[2] A. Douady and J. H. Hubbard, On the dynamics of polynomial-like mappings, Ann. Sci. Ec. Norm. Sup 18(1985), pp 287-344.

[3] A. Chademan and A. Zireh, Extension of the Douady-Hubbard's Theorem on Connecteness of the Mandelbrot set to Symmetric Polynomials, Bull. of Iranian Math. Soc. 31, no. 1 (2005), 77-84.

[4] J. H. Hubbard, Local connectivity of Julia sets and bifurcation loci: three theorems of J.-C. Yoccoz, pp 467-511 of "Topological Methods in Modern Mathematics", ed. Goldberg and Phillips,Publish or Perish 1993.

[5] M. Lyubich and J. Kahn, Local connectivity of Julia sets for unicritical polynomials IMS at Stony Brook Preprint 2005/03.

[6] J. Milnor, Dynamics in one complex variable, American Mathematical Society, Providence (2000).

[7] J. Milnor, Geometry and dynamics of quadratic rational maps. Experiment. Math. 2 (1993), no 1, pp 37-83.

[8] M. Rabii, Local connectedness of the Julia set of the family $z^{m}\left(z^{n}-b\right)$, Ergodic Theory and Dynamical System 18 (1998), pp 457-470.

[9] D. Schleicher, On Fibers and Renormalization of Julia Sets and Multibrot Sets,Stony Brook IMS 1998.

[10] N. Steinmetz, Rational Iteration. Complex Analytic Dynamical System, de Gruyter Studies in Mathematics, 16. Walter de Gruyter Co., Berlin, 1993.

Received: November 21, 2006 